\title{
Evidence of Absence in the D-HEALTH Trial: A Bayesian Reanalysis
}

\author{
Eric-Jan Wagenmakers \& Quentin F. Gronau
}

Affiliation: Psychological Methods Unit, University of Amsterdam

Email of corresponding author: EJ.Wagenmakers@gmail.com

Word count: 604

Address for correspondence:

Eric-Jan Wagenmakers

Department of Psychological Methods, room G 0.29

University of Amsterdam, Nieuwe Achtergracht 129B

Letter: PO Box 15906, 1001 NK Amsterdam

Parcel: Valckenierstraat 59, 1018 XE Amsterdam 
The D-HEALTH trial ${ }^{1}$ concluded that "Administering vitamin $D_{3}$ monthly to unscreened older people did not reduce all-cause mortality". Indeed, the results were opposite to those expected: deaths were slightly more prevalent in the vitamin D group (562 of 10,662 patients: $5.27 \%$ ) than in the placebo group (538 of 10,653 patients: $5.05 \%$ ). It appears that the data offer evidence of absence of a treatment effect, but from non-significant $p$-values and confidence intervals one can obtain no more than an imprecise, intuitive, and subjective impression of the strength of such evidence.

Here we quantify the degree to which the data from the D-HEALTH trial, considered in isolation, undercut the hypothesis that a vitamin $\mathrm{D}$ regimen lowers all-cause mortality. We conducted Bayesian logistic regression ${ }^{2,3}$ and contrasted two rival models. Under the no-effect model $H_{0}$, (i.e., vitamin $D$ is ineffective) the log odds ratio equals $\psi=0$; under the positive-effect model $H_{+}$(i.e., vitamin $D$ reduces mortality), $\psi$ is assigned a positive-only normal prior distribution $\mathrm{N}_{+}(\mu, \sigma)$, reflecting the directionality of the expected effect.

A default analysis (i.e., $\mu=0, \sigma=1$ ) reveals strong evidence for $\mathrm{H}_{0}{ }^{4}$ Specifically, the observed data are about 26.67 times more likely under $\mathrm{H}_{0}$ than under $\mathrm{H}_{+}$. This evidence would increase the probability for $\mathrm{H}_{0}$ from 0.50 before having seen the data to about 0.96 after having seen the data. Figure 1 shows that the evidence for $\mathrm{H}_{0}$ is strong ${ }^{4}$ (i.e, larger than 10) for a wide range of prior distributions based on combinations of $\mu$ in $[0,0.3]$ and $\sigma$ in $[.25,1]$. Only when $\mu$ and $\sigma$ are both small is the evidence not compelling - a natural consequence of specifying $\mathrm{H}_{+}$in such a way that it makes predictions that are highly similar to those of $\mathrm{H}_{0}$.

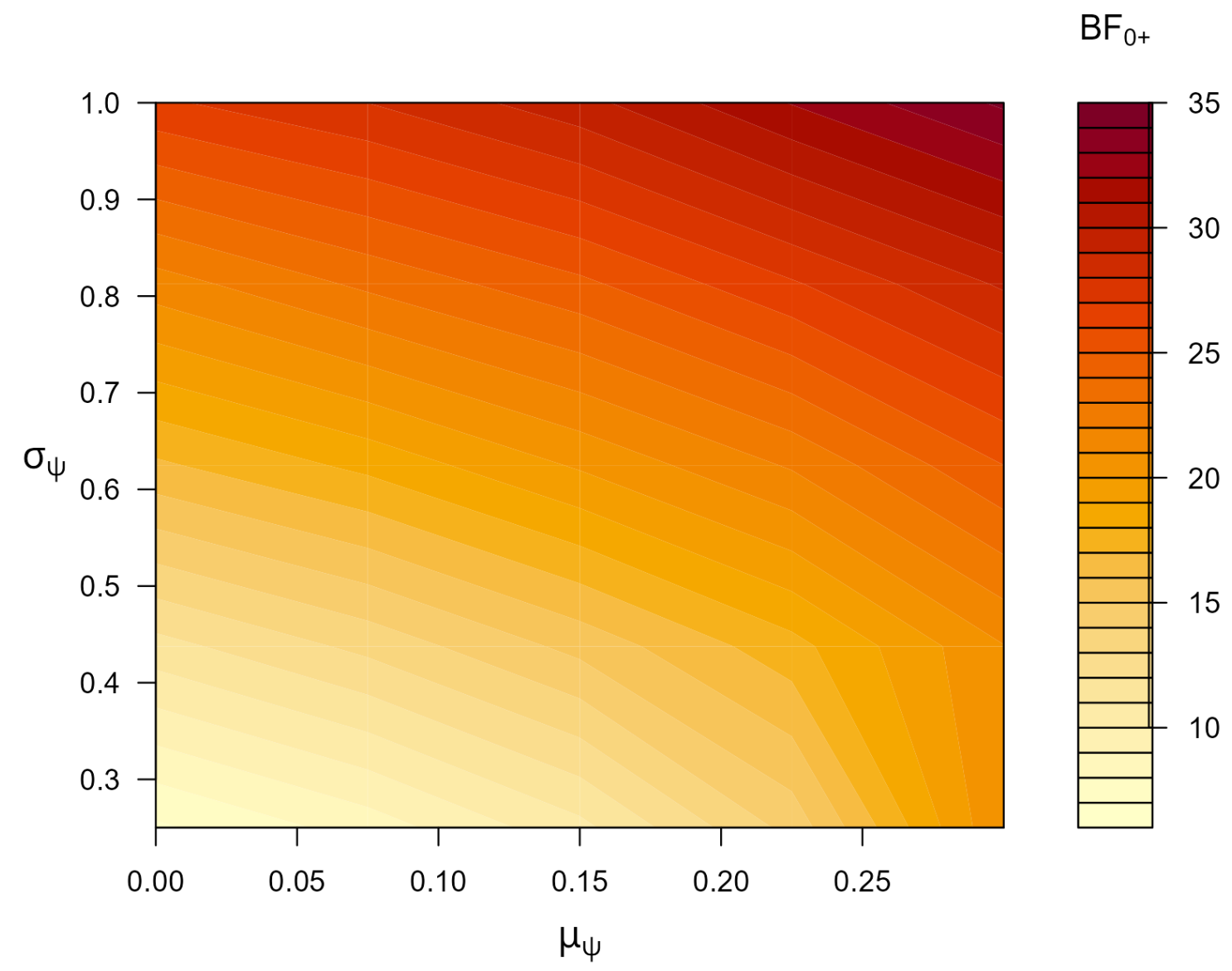


Figure 1. Across a wide range of prior distributions for the log odds ratio under $\mathrm{H}_{+}$, there is considerable evidence for the no-effect model $H_{0}$ over the positive-effect model $H_{+}$. Figure from JASP (jasp-stats.org).

Disregarding $\mathrm{H}_{0}$ for the purpose of parameter estimation, Figure 2 shows the prior and posterior distribution for the log odds ratio under a nondirectional alternative hypothesis. The posterior mass is concentrated near a log-odds ratio of 0 , with most mass on negative values (corresponding to a health detriment of vitamin D).

$$
\text { median }=-0.044
$$

95\% Cl: $[-0.165,0.077]$

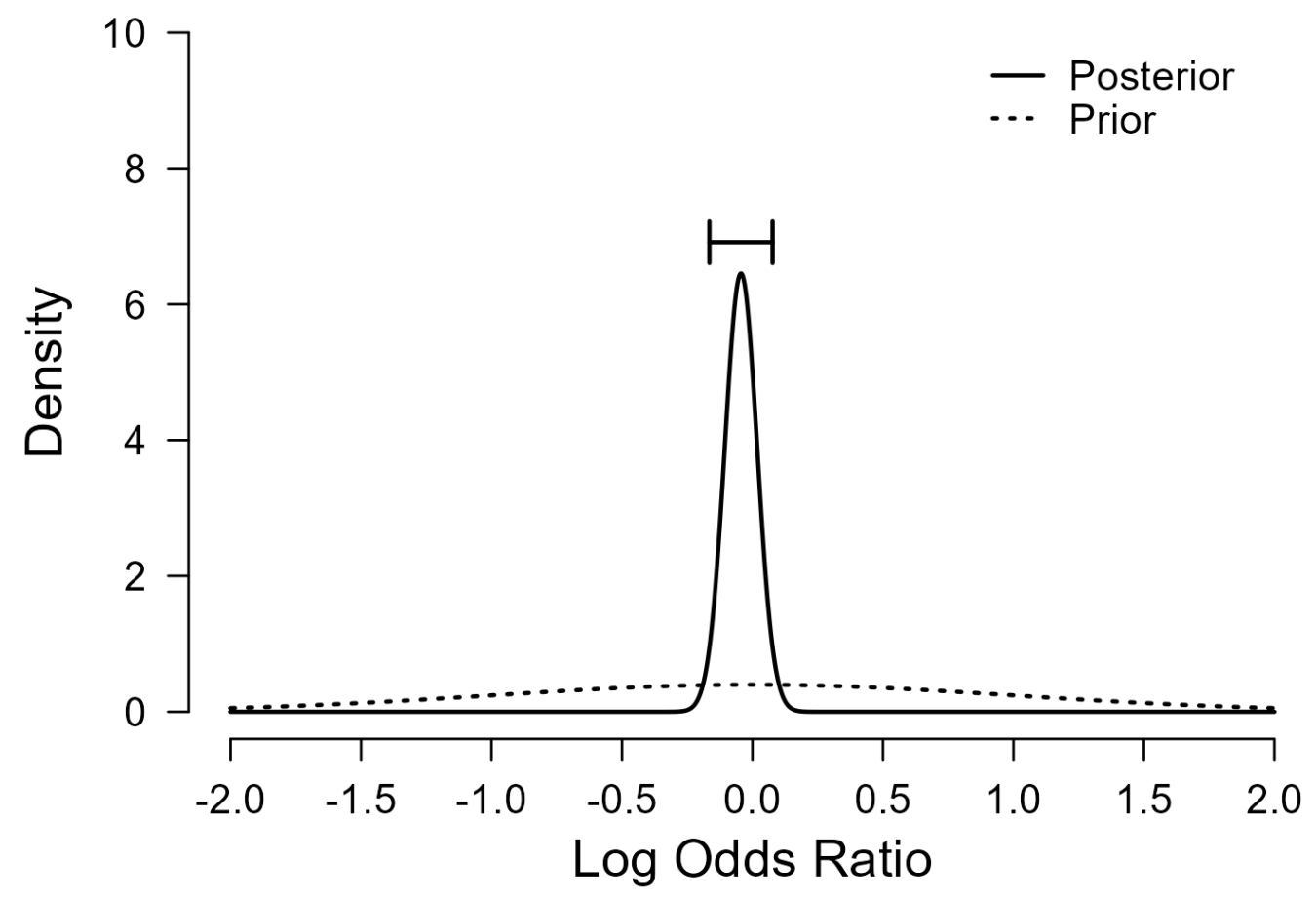

Figure 2. Prior and posterior distribution of the log odds ratio $\psi$ for the effect of vitamin $D$ on all-cause mortality. Under a nondirectional standard normal prior distribution, it is $95 \%$ probable that the true value of $\psi$ falls between -0.165 and 0.077 . Figure from JASP (jasp-stats.org).

The same information is presented in Figure 3, but now on the probability scale. Compared to the posterior distribution for mortality in the placebo group (p2), the posterior distribution for mortality in the vitamin D group (p1) is shifted towards larger chances, although the extent of this shift is very small. 


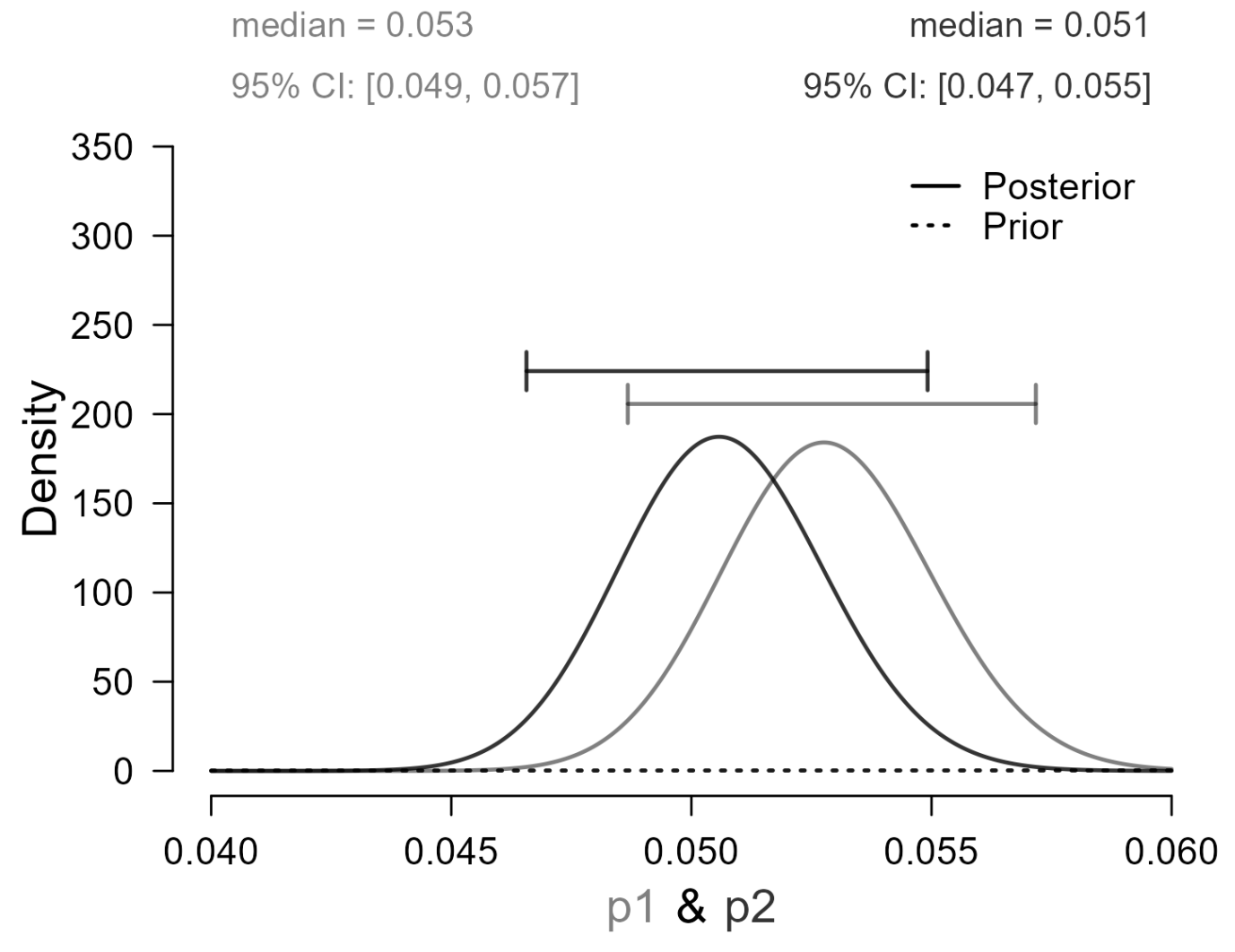

Figure 3. The difference between the two posterior distributions indicates the size of the effect on the probability scale. The gray " $p 1$ " and the black " $p 2$ " indicate the all-cause mortality probability in the vitamin D group and the placebo group, respectively. Figure from JASP (jasp-stats.org).

In sum, the data from the D-HEALTH trial strongly increase the plausibility that a vitamin D regimen is ineffective in lowering all-cause mortality. If a beneficial effect does exist, it is likely to be very small. These analyses, easily conducted in JASP (jasp-stats.org) or R (https://CRAN.R-project.org/package=abtest), arguably provide a more detailed perspective that supplements the reported $p$-values and confidence intervals. 


\section{References}

1. Neale RE, Baxter C, Romero BD, McLeod DSA, English DR, Armstrong BK, Ebeling PR, Hartel G, Kimlin MG, O'Connell R, van der Pols JC, Venn AJ, Webb PM, Whiteman DC, Waterhouse M. The D-Health Trial: a randomised controlled trial of the effect of vitamin D on mortality. Lancet Diabetes Endocrinology 2022; 10: 120-28.

2. Kass RE, Vaidyanathan SK. Approximate Bayes factors and orthogonal parameters, with application to testing equality of two binomial proportions. Journal of the Royal Statistical Society: Series B (Methodological) 1992;54:129-44.

3. Gronau QF, Raj K. N. A., Wagenmakers EJ. (2021). Informed Bayesian inference for the A/B test. Journal of Statistical Software, 100, 1-39.

4. Jeffreys, H. Theory of Probability. 1st ed. Oxford University Press, Oxford, UK, 1939. 